alien intelligences and a chapter on how and why we perceive the Universe the way we do. These ideas all deal with known laws of physics but impinge directly on the mathematical world in that they question whether some things are knowable or might never be knowable. In the case of modelling the Universe, it might be that only by looking at the real Universe can we find out the answer to certain questions, and the traditional computational shortcuts of theoretical physics simply do not work. It might be that Godel's incompleteness theorem prevents us from describing everything. Or in the case of extra-terrestrial intelligence, it might be that things are simply so alien that we are incapable of recognizing things for what they are.

But back to physics, there is a chapter on the origin of mass where some interesting ideas are introduced. Here, however, the picture presented could easily change dramatically with the anticipated switch-on of the
Large Hadron Collider (LHC) at CERN next year. At some level, the origin of mass is associated with the hypothetical Higgs particle and the LHC is expected to discover it, if it indeed exists.

I have left till the end one topic that is particularly fascinating to anyone who has studied quantum mechanics. Given that quantum mechanics holds at the most fundamental level it always seems puzzling that the world is to a high degree of accuracy classical. This conundrum is always attributed in textbooks to the mysterious phenomenon of the collapse of the wavefunction, but this explanation is deeply unsatisfactory as it posits that there are really two incompatible descriptions of physics, one for describing how things behave and the other, rather mystical, describing observations. One of the great achievements in the foundations of quantum mechanics in the last fifteen years or so is 'decoherence', the idea that as a result of interactions with a complicated environment, a quantum system effectively behaves classically. There is an excellent treatment of this material here.

The book concludes with a short list of fundamental and fascinating questions. No doubt a future work will discuss these further. As a whole the book is an excellent introduction to many of the fascinating questions being addressed in theoretical physics and cosmology today. It is stimulating in the sense that readers are being encouraged to go and read or inquire more or perhaps pursue their own research in these areas, and it is extraordinary that questions that would have been regarded as theological not so long ago are now being answered by science. To those who want to learn about such questions I whole-heartedly recommend this book.

\section{Malcolm J. Perry}

Malcolm J. Perry is in the Department of Applied Mathematics and Theoretical Physics, Centre for Mathematical Sciences, University of Cambridge, Wilberforce Road, Cambridge CB3 OWA, UK.

\title{
Back to a space future
}

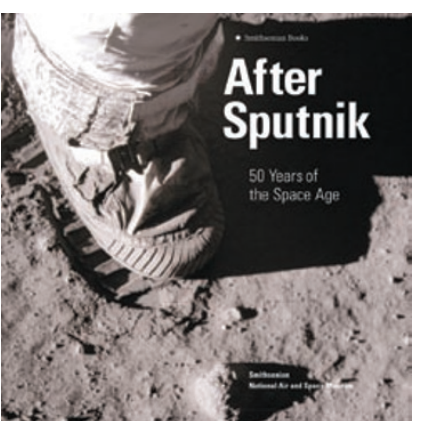

AFTER SPUTNIK: 50 YEARS OF THE SPACE AGE EDITED BY MARTIN COLLINS

Smithsonian Books/Harper Collins: 2007. 256 pp. \$35.00.
Describing the era we live in today as the 'Space Age' has an uncomfortable, slightly bombastic feel to it. How quickly things change: even as a child growing up in the 1980 s, I seem to remember the phrase being used as a tag with pretensions to historical validity, rather like 'Roman times' or 'the Victorian era'.

Then came space flight's loss of innocence in the 1986 Challenger disaster, and shortly after that, the end of the Cold War, which robbed the space race of much of its political rationale. Although After Sputnik provides an illuminating account of manned spaceflight right up to the present, its core harks back to a halcyon 'heroic age' of Gagarin and Glenn, and Armstrong and Aldrin. Its medium is artefacts from the collection of the Smithsonian National Air and Space
Museum in Washington DC, and attendant expository texts.

Understandably, the story thus told has an American bias. But it is a fascinating story nonetheless: starting with the thermos flask with which Robert H. Goddard first demonstrated the principle of liquid rocket-propellants in the 1920s, we pass by exhibits such as Soviet stamps commemorating Yuri Gagarin's first Earth orbit, the spacesuit worn by Neil Armstrong on the Apollo 11 Moon mission, the carbon dioxide filters extemporized in mid-flight to save Apollo 13, and the massive hulk of NASA's first space shuttle, Enterprise.

But there's also the unexpected, the whimsical and the poignant. A tube of sour green cabbage soup provided a taste of home for Soviet astronauts of the 1960s. The pickled remains of Anita, an orb-weaver spider, are a testament to life's adaptability in space: after an initial short period of forgivable disorientation on a 1973 Skylab flight, she wove webs perfectly suited to microgravity, using silk 20 per cent finer than on Earth.

Last but not least, a couple of exhibits provide answers to that most trivial and yet most fundamental of questions - what do you do when you are caught short in space? Such gems are interspersed with cultural artefacts, from Star Trek, Star Wars, Babylon 5 and the like, to make for a satisfyingly rounded scientific and social history of the iconic race for space.

Richard Webb

Richard Webb is a News \& Views editor for Nature. 